Nevertheless, in many children and adults ascaris infection is symptomless, an ideal host-parasite relationship apparently having been established.

${ }^{1}$ Edington, G M, and Gilles, H M, Pathology in the Tropics, 2nd edn. London, Arnold, 1976.

2 Denzler, T B, and Gunning, J-J, Archives of Internal Medicine, 1976, 136, 1044

${ }^{3}$ Crane, P S, Pak, Y H, and Lee, H K, Annals of Surgery, 1965, 162, 34.

${ }^{4}$ Gilles, H M, British Medical fournal, 1976, 2, 1314.

\section{Mucocutaneous lymph node syndrome: a new disease}

In 1961 Japanese physicians began to see cases of a mysterious new disease affecting infants and young children. Kawasaki tentatively named it the mucocutaneous lymph node syndrome (MLNS) of infancy, and in 1967 he reported his experience of 50 cases in a Japanese journal. His paper was quickly followed by more reports, also in Japanese, and in 1970 the Japanese Government set up a research committee to examine the clinical manifestations of MLNS, its pathogenesis, and its apparently increasing incidence. By 1973 this committee had already received details of 4000 cases.

Once the disease was described in English language journals ${ }^{12}$ cases began to be reported from outside Japan: in Hawaii, ${ }^{3}$ Korea, ${ }^{4}$ and Greece $^{5}$ and eventually in white children in the United States. Recently Darby and Kyong ${ }^{6}$ detailed a typical case in a $2 \frac{1}{2}$-year-old white American girl and told of the identification of cases in South Carolina once groups of practitioners there had seen descriptions and photographs of the clinical signs.

Kawasaki's name for the disorder has stuck. The clinical picture of MLNS is striking, and it really seems to be a new disease rather than the result of clustering of unusual or selected features of other disorders. The Japanese research committee found that most patients were under 5 years and that half were less than 2 . Typically, a child becomes acutely ill with fever, dry lips, red mouth, and undue protuberance of the tongue papillae. On the third or fourth day a generalised polymorphous exanthem appears and becomes confluent, bright red in a glove-and-sock distribution. The skin becomes indurated and oedematous and eventually desquamates from the fingers and toes. Cervical lymphadenitis is common, but the nodes do not suppurate. Nonspecific acute phase reactants in the blood are all positive-a polymorphonuclear leucocytosis, raised ESR, raised $\alpha_{2}$-globulin, and positive $C$ reactive protein.

Recovery is usual, during the second to fourth weeks, but the course may be complicated by aseptic meningitis, arthralgia or arthritis, jaundice with a slight increase in serum transaminases, and myocarditis or cardiac infarction. Nevertheless, $1-2 \%$ of reported cases ended fatally: death was sudden in all and was caused by coronary artery thrombosis.

There is no response to antibiotics, and no treatment has so far been shown to have any effect on the course of the disease. No bacterial origin has been found; antistreptococcal antibody titres are low. No virus has been isolated, and complement fixation tests are negative. Recently, however, Hamashima has reported rickettsia-like bodies in biopsy specimens of skin or lymph node in 12 out of 23 patients. ${ }^{7}$ These bodies were found in the cytoplasma of macrophages, in endothelial cells of arterioles, and clustered inside the vascular lumina. Further studies are being made.

The pathological features of the fatal cases are distinctive and important. Of the 168 cases reported by Kawasaki four ended in sudden death. All the fatal cases have shown coronary artery thrombosis with aneurysm formation resembling that seen in infantile polyarteritis nodosa. ${ }^{8}$ The development of myocardial infarction and the angiographic abnormalities found in surviving cases suggest that this complication may cause permanent cardiac damage.

The differential diagnosis is from the infantile form of polyarteritis, Stevens-Johnson syndrome, and scarlet fever. MLNS affects a generally younger age group than the last two, though there is overlap, but, more importantly, the glowing redness and induration of the extremities seems to be a pathognomonic feature. Coronary artery lesions are exceptional in children's diseases (apart from infantile polyarteritis) and are not found in either Stevens-Johnson syndrome or scarlet fever.

So far it is unknown whether the disease is really new or only newly recognised, but it seems unlikely that such a striking entity could have evaded description until so recently. Probably, therefore, a cause will be found (as in other "new" diseases recently described, such as Lassa fever and kuru) and this may be infective. If so the change in incidence may be attributable to a change in the behaviour, distribution, or virulence of the causal organism. The speed with which cases have been reported from outside Japan as soon as the disease became more widely known suggests that medical practitioners in Britain should be aware of MLNS and on the look out for it. As the disease becomes better recognised epidemiological studies may help towards a solution.

${ }^{1}$ Kawasaki, T, et al, Pediatrics, 1974, 54, 271.

${ }^{2}$ Fetterman, G H, and Hashida, Y, Pediatrics, 1974, 54, 268.

3 Melish, M E, Hicks, R M, and Larson, E, Pediatric Research, 1974, 8, 427.

$4 \mathrm{Kim}$, J, Yeo, Y, and Lee, D B, Korea New Medical fournal, 1973, 16, 1157.

5 Valaes, T, Pediatrics, 1975, 55, 295.

${ }^{6}$ Darby, C P, and Kyong, C U, fournal of the American Medical Association, 1976, 236, 2295

7 Hamashima, Y, Kishi, K, and Tasaka, R, Lancet, 1973, 2, 42.

8 Kato, H, et al, Fournal of Pediatrics, 1975, 86, 892.

\section{Doctors and pressure groups}

Anyone who joins a pressure group has to be committed to the cause. Long hours need to be spent on committees, planning campaigns, marching, lobbying MPs, and bombarding the press with information; and only enthusiasts are prepared to give their time and energy on the scale required, often for no financial reward. So the partiality of campaigners should not be surprising, whether their target is smoking, Concorde, fluoridation, or experiments on animals. Their techniques have much in common with successful advocates, who choose their questions with care, and even when restricted by the rules of court can often lead a witness into making damaging admissions. Doctors should not be surprised when they are asked somewhat loaded questions on emotive topics such as abortion or euthanasia, and they should pause and think before answering - and perhaps refuse to answer at all if the question is of the familiar pattern of "Have you stopped beating your wife ? Answer yes or no."

In recommending an attitude of circumspection and caution we are not trying to turn the clock back to the bad old days when doctors never talked to the press. Indeed, so much good has come from easier relations with journalists, and so much current coverage of medical questions by the radio, television, and newspapers is fair that it is sad that any warning is needed. Yet there are occasions when doctors may find themselves lending their authority to distortions of their 